\title{
GADD45a Mediated Cell Cycle Inhibition Is Regulated By P53 In Bladder Cancer
}

This article was published in the following Dove Press journal: OncoTargets and Therapy

\begin{abstract}
Na Han, ${ }^{1,2, *}$ Fang Yuan, ${ }^{2,3, *}$ Peng Xian, ${ }^{2,3} *$ Nan Liu, ${ }^{2,3}$ Jianmin Liu, 4 Haiyan Zhang, ${ }^{1,2}$ Huayong Zhang, (iD ${ }^{5}$ Kai Yao, ${ }^{6}$ Gangjun Yuan (D) ${ }^{2,3}$

'Health Examination and Oncology Screening Center, Chongqing University Cancer Hospital, Chongqing 400030, People's Republic of China; ${ }^{2}$ Chongqing Key Laboratory of Translational Research for Cancer Metastasis and Individualized Treatment, Chongqing University Cancer Hospital, Chongqing 400030, People's Republic of China; ${ }^{3}$ Department of Urology, Chongqing University Cancer Hospital, Chongqing 400030, People's Republic of China; ${ }^{4}$ Department of Otolaryngology Head and Neck Surgery, People's Hospital of Deyang, Deyang 618000, People's Republic of China; ${ }^{5}$ Department of Thyroid and Breast Surgery, The Fifth Affiliated Hospital of Sun Yat-sen University, Zhuhai 519000, People's Republic of China; ${ }^{6}$ Department of Urology, Sun Yat-Sen University Cancer Center, State Key Laboratory of Oncology in South China, Collaborative Innovation Center for Cancer Medicine, Guangzhou, People's Republic of China
\end{abstract}

*These authors contributed equally to this work

Correspondence: Gangjun Yuan Department of Urology, Chongqing University Cancer Hospital, Chongqing 400030, People's Republic of China

Tel +862365075629

Email freeman17@I26.com

Kai Yao

Department of Urology, Sun Yat-sen University Cancer Center, State Key Laboratory of Oncology in South China,

Collaborative Innovation Center for

Cancer Medicine, Guangzhou 510060,

People's Republic of China

Tel +862087343840

Email yaokai@sysucc.org.cn
Background: Bladder cancer $(\mathrm{BC})$ is one of the most prevalent malignancies of the genitourinary system, yet the underlying mechanism of $\mathrm{BC}$ progression still remains unclear. Growth arrest and DNA damage-inducible 45 alpha (GADD45a) is a repressive gene implicated in cell cycle regulation, as well as in human cancers development. However, its role in $\mathrm{BC}$ remains to be determined.

Methods: First, quantitative real-time polymerase chain reaction (PCR) and Western blot assays were used to detect GADD45a expression in BC tissues and adjacent non-tumor tissues, as well as in bladder cancer cell lines, respectively. Then, cell counting kit-8 (CCK-8) assays, colony formation assays, and flow cytometry assays were used to measure the ability of cell growth, proliferation and cell cycle distribution. Lentiviral infection technology was used to increase gene expression, while siRNA interfering technology was used to knockdown gene expression. Finally, nude mice were used to construct tumor-burdened models in vivo by injecting tumor cells subcutaneously.

Results: PCR results showed that the level of GADD45a mRNA and protein levels were lower in $\mathrm{BC}$ tissues than in adjacent normal tissues. After increasing GADD45a expression, both the ability of growth and proliferation of $\mathrm{BC}$ cells were seriously impaired. Additionally, the upregulation of GADD45a expression resulted in BC cell cycle in G2/M and $\mathrm{S}$ phases in a p53-regulated pathway.

Conclusion: GADD45a-mediated cell cycle inhibition is regulated by p53 in bladder cancer cells.

Keywords: GADD45a, bladder cancer, cell cycle, p53, proliferation

\section{Introduction}

Bladder cancer (BC) is still one of the most risky malignancies affecting the genitourinary system, especially in Chinese people, with an increased risk of both mortality and mobility according to a clinical data in $2015 .^{1}$ It is reported that there were 74,690 new BC cases, leading to 25,580 deaths in 2014. ${ }^{2}$ Much efforts has been devoted to studying BC progression. However, the molecular mechanism still remains to be determined. Thus, there is an urgent need to explore how $\mathrm{BC}$ develops and progresses.

Our previous work showed that growth arrest and DNA damage-inducible 45 alpha gene (GADD45a) may play a repressive role in $\mathrm{BC}$ cell proliferation by delaying cell cycle progression in the G2/M phase. ${ }^{3}$ GADD45a was first found and defined by Fornace et al in 1989 when researchers found that some mRNAs were increased after exposure to a variety of exogenous and endogenous stresses associated with growth arrest, including ultraviolet (UV) radiation. ${ }^{4}$ GADD45a belongs 
to a highly conserved three-gene GADD family with two other members GADD45b and GADD45g. These genes were first cloned from Chinese hamster ovary $(\mathrm{CHO})$ cells after exposure to UV radiation and functioned as a subset of transcription factors. ${ }^{5-7}$ GADD45a protein localizes within the nucleus and interacts with $\operatorname{cdc} 2 /$ cyclinB1 kinases to inhibit cell cycle progression in the $\mathrm{G} 2 / \mathrm{M}$ and S phase. ${ }^{8-10}$ In addition, GADD45a is involved in DNA damage, apoptosis, cell injury, and other growth regulatory processes.

Because of its repressive activity in cell proliferation, GADD45a is believed to have a negative role in carcinogenesis. Hollander et al reported that knockdown of GADD45a in a mouse lung cancer model led to higher malignancy tumors and an increased risk of multiple tumor types. ${ }^{11}$ Also, GADD45a was found to suppress the tumor angiogenesis by downregulating VEGFa expression via blocking the mTOR/STAT3 pathway. ${ }^{12}$ However, the role of GADD45a in BC has not yet been explored.

Thus, in this study, we investigated the expression of GADD45a in BC tissues and cells to reveal its potential role in $\mathrm{BC}$ progression using a series of in vivo and in vitro experiments.

\section{Materials And Methods}

\section{BC Tissues}

Two groups of six paired fresh MIBC tissues and adjacent non-tumor tissues from the same patient were stored in liquid nitrogen for Western blot and quantitative RT-PCR assays. Clinical data of the six patients are shown in Table 1. All samples were classified according to the 2010 American Joint Committee on Cancer TNM classification. All BC tissues were histologically identified to be urothelial carcinomas. The Medical Ethics Committee of The Sun Yat-Sen University Cancer Center approved this study, and all patients provided their consent to use their clinical specimens.

\section{Cell Culture}

UCB cell lines, T24, BIU, UMUC3, and 5637, were obtained from the American Type Culture Collection in 2003. Stocks were prepared after passage 2 and stored in liquid nitrogen. These cell lines were authenticated by the China Center for Type Culture Collection of Wuhan University and Mycoplasma testing was done by the authors before initiating this study. All experiments were performed with cells of $<8$ passages. Cell lines 293-T and SV-HUC-1 were purchased from the Institute of Biochemistry and Cell Biology, Shanghai Institutes for Biological Sciences, Chinese Academy of Sciences. BC cell lines and normal urothelial cell line SV-HUC-1 were cultured in RPMI 1640 media, while UMUC3 and 293-T cell lines were cultured in DMEM media. All media were supplemented with $10 \%$ fetal bovine serum (FBS), penicillin $(100 \mathrm{U} / \mathrm{mL})$ and streptomycin $(100 \mu \mathrm{g} / \mathrm{mL})$ (all three materials were from Gibco, ThermoFisher, USA) at $37{ }^{\circ} \mathrm{C}$ in $5 \% \mathrm{CO}_{2}$ atmosphere.

\section{Cell Viability Assay}

Cell viability was measured according to the manufacture's protocol of CCK- 8 (cell counting kit- 8 ) reagent (Dojindo, Kumamoto, Japan). Briefly, a total of $5 \times 10^{3}$ cells/well were seeded in 96-well plates to incubate for cell viability measurements at different time points of 12 h, 24 h, 36 h, 48 h, 60 h, and 72 h. The CCK-8 kit was added per well to co-incubate for $2-4 \mathrm{~h}$ before detection. Absorbance was measured at $450 \mathrm{~nm}$ in each well with a microplate spectrophotometer (SpectraMax M5; Molecular Devices, Sunnyvale, CA, USA).

\section{Colony Formation Assay}

Approximately 500 cells were seeded into each well in a six-well plate and incubated for 5-7 days. Colonies were fixed with methanol for $30 \mathrm{~min}$ and stained with $0.1 \%$ crystal violet for $1 \mathrm{~h}$.

Table I Clinical Information Of 6 Cases Whose Tissues Were Used For qRT-PCR And Western Blot Assay

\begin{tabular}{|l|l|l|l|l|l|}
\hline NO. & GENDER & AGE (YEAR) & STAGE & GRADE & SMOKING \\
\hline I & M & 57 & T2 & G3 & Yes \\
2 & M & 63 & T2 & G3 & Yes \\
3 & F & 65 & T3 & G3 & No \\
4 & F & 47 & T2 & G3 & No \\
5 & M & 72 & TI & G3 & Yes \\
6 & M & 67 & T2 & G3 & \\
\hline
\end{tabular}

Abbreviations: $M$, male; F, female. 


\section{Cell Cycle Analysis}

Determined cells were harvested and then washed with cold PBS for three times, followed by fixating the cells in chilled $70 \%$ ethanol overnight at $4 \square$. A cell cycle analysis kit (Beyotime, Shanghai, China) was used to measure cell cycle distribution following the manufacture's protocol. The cell cycle determination was performed using a flow cytometry system (MoFlo XDP, Beckman Coulter, CA, USA).

\section{Western Blot Analysis}

Determined cells were harvested and lysed to obtain protein extracts for further electrophoresis analysis. For fresh and frozen $\mathrm{BC}$ tissues, they were ground in liquid nitrogen and then lysed for obtaining protein lysates. All protein extracts were separated on $10 \%$ sodium dodecyl sulfate polyacrylamide gel electrophoresis (SDS-PAGE) gels, and electrotransferred to a polyvinylidene fluoride (PVDF) membrane at $250 \mathrm{~mA}$ for $2 \mathrm{~h}$ at room temperature. Then the membrane was blocked in $5 \%$ bovine serum albumin or defatted milk for $1 \mathrm{~h}$ at room temperature and incubated overnight at $4^{\circ} \mathrm{C}$ with primary antibodies (GADD45a, Tubulin- $\alpha$ and GAPDH antibodies were from Proteintech Group Inc., Rosemont, IL, USA; p53, cdc2, cyclinB1, CDK2, CDK4 and cyclinD1 were from Cell Signaling Technology, Danvers, MA, USA;). The membrane was washed three times with phosphate-buffered saline (PBS) containing $0.1 \%$ Tween (PBST), followed by incubation with a secondary antibody for $1 \mathrm{~h}$ at room temperature. The signal was measured using an enhanced chemiluminescence (ECL) detection system (Tanon, Shanghai, China).

\section{Quantitative Real-Time Polymerase Chain Reaction (qRT-PCR) Assay}

The frozen tissues were ground in liquid nitrogen, and total RNA was extracted using TRIzol reagent (Invitrogen Life Technologies, USA) according to the manufacturer's recommendations. Approximately $1 \mu \mathrm{g}$ of RNA was used in firststrand cDNA synthesis using random primers. A $15 \mu \mathrm{L}$ reaction system, which included the GADD45a specific primers, cDNA, and SYBR Green PCR mixture (Applied Biosystems), was prepared for amplification of the GADD45a cDNA. The qRT-PCR reaction conditions were as follows: initial denaturation at $95^{\circ} \mathrm{C}$ for $30 \mathrm{~s}$, followed by annealing at $55^{\circ} \mathrm{C}$ for $1 \mathrm{~min}$, and extension for $1 \mathrm{~min}$ at $72^{\circ} \mathrm{C}$, for a total of 30 cycles. The process was performed in a triplicate on an ABI Prism 7000 sequence detection system (Applied Biosystems, Foster City,
CA, USA). The relative level of gene expression was expressed as $\Delta \mathrm{Ct}=\mathrm{Ctgene}-\mathrm{Ctreference,}$ and the $2-\Delta \Delta \mathrm{Ct}$ method was used to calculate the fold change of gene expression. Tubulin- $\alpha$ was used as a control and for normalization. The primer sequences are as follows: for GADD45a, forward, 5'-TCGTGAAATGGAAGGGATGG-3'; reverse, 5'-AGGTT TTGGGCTTGGGTC-3'; and for GAPDH, forward, 5'-CGG AGTCAACGGATTTGGTCGTAT-3' and reverse, 5'AGCC TTCTCCATGGTGGTGAAGAC-3'.

\section{Overexpression Experiment}

A lentivirus for GADD45a and p53 overexpression was purchased from HanBio (Shanghai, China). The virus vector was pHBLV-CMVIE-Zs Green-T2A-Puro. The final virus titer of overexpressing lentivirus and negative control virus was $2 \times 10^{8} \mathrm{PFU} / \mathrm{mL}$. T24 cells were transfected with the lentivirus to overexpress GADD45a and p53 premixed with lipofectamine 2000 (Invitrogen, USA). Stable cells were screened with puromycin (Gibco, Invitrogen, Darmstadt, Germany) at the concentration of $2 \mu \mathrm{g} / \mathrm{mL}$ for two weeks.

\section{Xenograft Assay}

Four-week-old Balb/c female nude mice were purchased from Charles River Laboratories (Beijing, China). The Animal Ethics Committee of Sun Yat-sen University, Guangzhou, China approved all animal experiments performed in this study. The welfare of the animals was guaranteed under the criteria of the "Laboratory Animal Management Regulations in China". Mice were randomly divided into 2 groups with 6 mice. A total of $5 \times 10^{5}$ T24 cells (control and overexpressing-GADD45a RNA) were subcutaneously inoculated into the right flank of the mice. The weight and volume of the tumors were determined at the end of the study. The following formula was used to measure tumor volume: Tumor volume $=1 / 2 \mathrm{~L} \times \mathrm{W}^{2}$, where $\mathrm{L}$ stands for the length and $\mathrm{W}$ is the width. All animal experiments were approved by the Animal Ethics Committee of Sun Yat-sen University cancer center.

\section{Results \\ GADD45a Expression In BC And Adjacent Non-tumor Tissues}

To determine the expression of GADD45a in BC, fresh tumor tissues and adjacent normal tissues were collected to obtain cellular proteins for further Western blot and quantitative PCR analysis. As shown in Figure 1A, GADD45a protein levels in $\mathrm{BC}$ tissues were lower than levels in the 


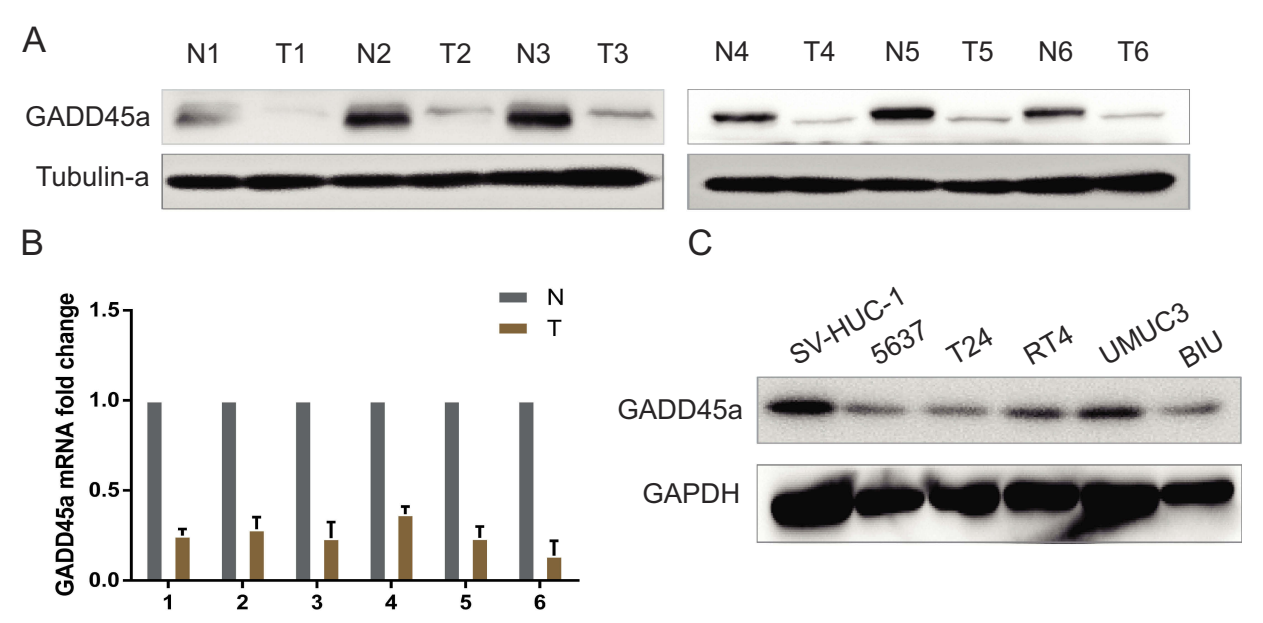

Figure I GADD45a expression in bladder cancer (BC). (A) Western blot analysis showed that GADD45a protein was downregulated more in BC tissues than in adjacent non-tumor tissues. (B) The result of quantitative real-time PCR detection showed that the level of GADD45a mRNA was significantly lower in BC tissues than adjacent normal tissues. Histograms display the mean number of colonies, and the number of colonies was shown as the mean \pm SD of three independent experiments. (C) Western blot assay in BC cell lines found that GADD45a was downregulated in BC cell lines comparing to normal urothelial SV-HUC-I cells.

Abbreviations: $\mathrm{T}$, tumor tissues; $\mathrm{N}$, normal tissues.

adjacent normal tissues. Subsequent qRT-PCR assay in these paired tissues revealed that GADD45a mRNA was decreased in tumor tissues when compared to the adjacent non-tumor tissues $(0.252 \pm 0.063$ vs. $1, \mathrm{p}<0.001$. Figure $1 \mathrm{~B})$. Both assays indicated that GADD45a was down-regulated in BC tissues.

\section{GADD45a Protein Expression In BC Cell Lines}

Because of GADD45a expression changes between BC tissue and surrounding tissues, we also characterized GADD45a expression changes in between several BC cell lines and the normal urothelial cell line SV-HUC-1. As expected, GADD45a was downregulated in BC cells compared to SV-HUC-1 cells (Figure 1C).

\section{Upregulation Of GADD45a Inhibits BC \\ Cell Growth And Proliferation}

To determine the role of GADD45a in BC cell growth, stable highly expressing GADD45a T24 cells were constructed. The CCK-8 kit was applied to detect the growth rate of $\mathrm{BC}$ cells. As shown in Figure 2A, increasing GADD45a expression decreased $\mathrm{BC}$ cell growth. In addition, it led to significantly fewer cell colonies than the control in two cell lines T24 and 5637 (T24, p = 0.032; $5637, \mathrm{p}=0.027$ ) (Figure 2B). Theses results indicated that upregulation of GADD45a impairs BC growth and proliferation.

\section{Upregulation Of GADD45a Inhibits BC Cell Cycle Progression In G2/M And S Phase}

Since reports indicate that GADD45a interacts with cdc2/ cyclinB1 kinases to delay cell cycle progression in the G2/ $\mathrm{M}$ and $\mathrm{S}$ phase, ${ }^{9,10}$ we tested whether GADD45a has a similar inhibitory effect on the BC cell cycle using a flow cytometry assay. Results indicated that upregulation of GADD45a resulted in cell cycle arrest in the G2/M and $S$ phase by increasing the percentage of cells in the $\mathrm{G} 2$ and $\mathrm{S}$ phase, and decreasing the percentage of cells in the G1 phase (Figure 3A). Meanwhile, G2-M transition related kinases cdc2 and cyclinB1 were dramatically decreased, whereas G1-S transition related CDK2, CDK4 and cyclinD1 were unchanged (Figure 3B).

\section{GADD45a Mediated BC Cell Cycle Inhibition Is P53-regulated}

GADD45a was the first described stress gene that is transcriptionally regulated by $\mathrm{p} 53$, a well-known repressive gene that is commonly downregulated in many phenotypes of human cancers. ${ }^{13}$ This information suggests that p53 may play a regulatory role in GADD45a-induced cell cycle inhibition. ${ }^{14}$ We found that p53 was downregulated in T24 cells compared to normal SV-HUC-1 cells (Figure 3C). Deletion of p53 would decrease GADD45a expression in SV-HUC-1 cells (Figure 3D). Nevertheless, increasing p53 
A T24

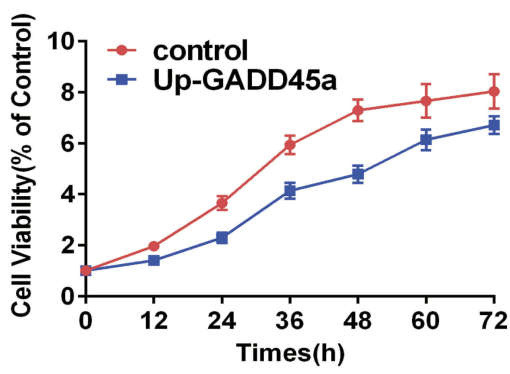

B
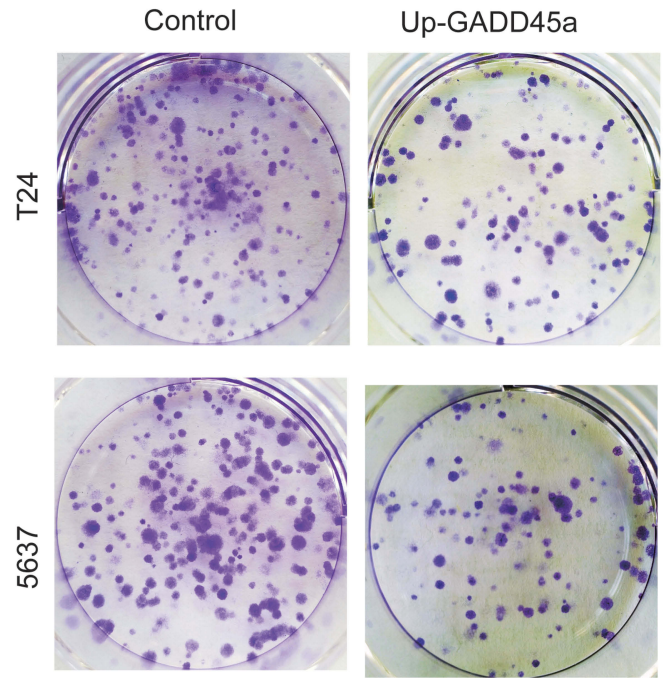

5637
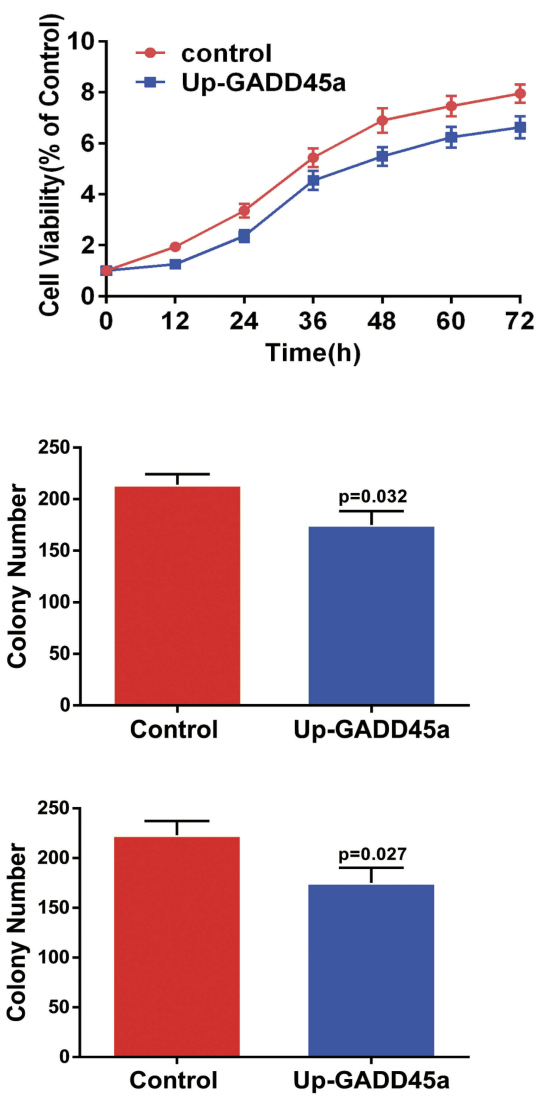

Figure 2 Upregulation of GADD45a inhibits BC cell growth and proliferation. (A) CCK-8 kit was used to measure the growth ability of BC cells and the results showed that the ability of BC T24 and 5637 cells with GADD45a upregulation was obviously inhibited compared to control cells. (B) A cell colony forming experiment was performed to detect the ability of BC cell proliferation. After upregulating GADD45a expression in T24 and 5637 cells, fewer colonies were formed in both cell lines $(P=0.032,0.027$ respectively). Histograms display the mean number of colonies, and the number of colonies was shown as the mean \pm SD of three independent experiments, $P<0.05$ represents significance.

expression has contributed to elevated GADD45a expression, as well as to similar effects on cell cycle distribution like increasing GADD45a expression (Figure 3E and F). Overall, these results suggest that $\mathrm{p} 53$ positively regulates GADD45a expression and GADD45a-mediated cell cycle inhibition.

\section{Upregulation Of GADD45a Inhibits BC Cell Growth In Vivo}

To validate the inhibitory role of GADD45a in BC cell proliferation, tumor burdened mice were constructed by injecting BC cells subcutaneously. The results showed that mice with highly expressing GADD45a cells generated smaller tumors than control mice (Figure 4A). The volumes of tumors, as well as the weight of mice, were clearly recorded every 2 days after injection of BC cells (Figure 4B and C). At the end of the study, mice were sacrificed by cervical dislocation, followed by isolation of the tumors to count tumor volumes and weight (Figure 4D and E). The result showed that the volumes of tumors in mice injected with highly expressing GADD45a cells were significantly smaller than the control $(137.86 \pm 7625$ vs. $845.57 \pm 201.37, \mathrm{p}<$ $0.001)$, The tumor weights $(314.83 \pm 59.57$ vs. $527.83 \pm$ $10.64, \mathrm{p}<0.001$ ) were also significantly smaller than the control. These results indicated that upregulating GADD45a significantly inhibits tumor growth in vivo.

\section{Discussion}

A series of cellular responses with regulators and effectors are involved in responding to genotoxic stress including genotoxic, physiological, and oncogenic stimuli. Evidence indicates that GADD45a plays a critical role as a sensor in cellular stress responses. ${ }^{15}$ In addition, our previous work suggested a repressive role of GADD45a in BC cell cycle 
A
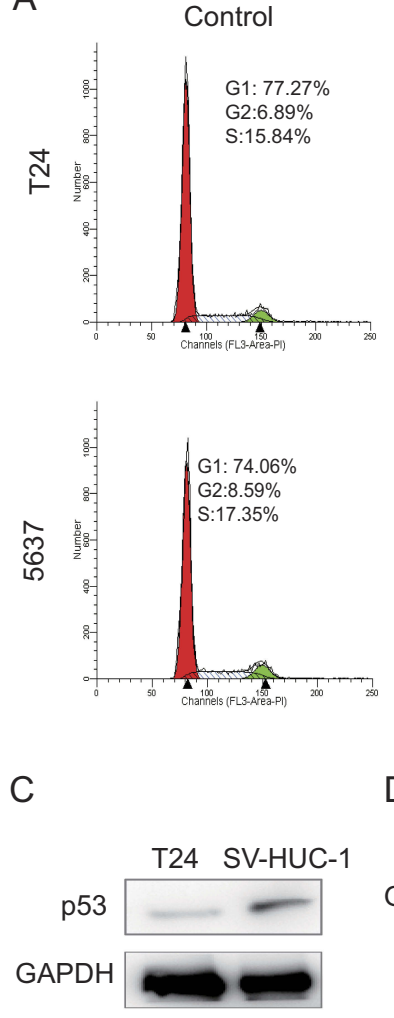

$\mathrm{F}$

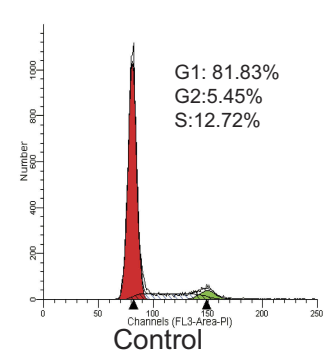

Up-GADD45a
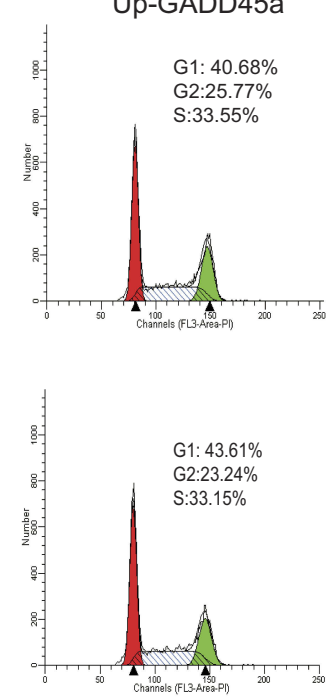

$\mathrm{D}$
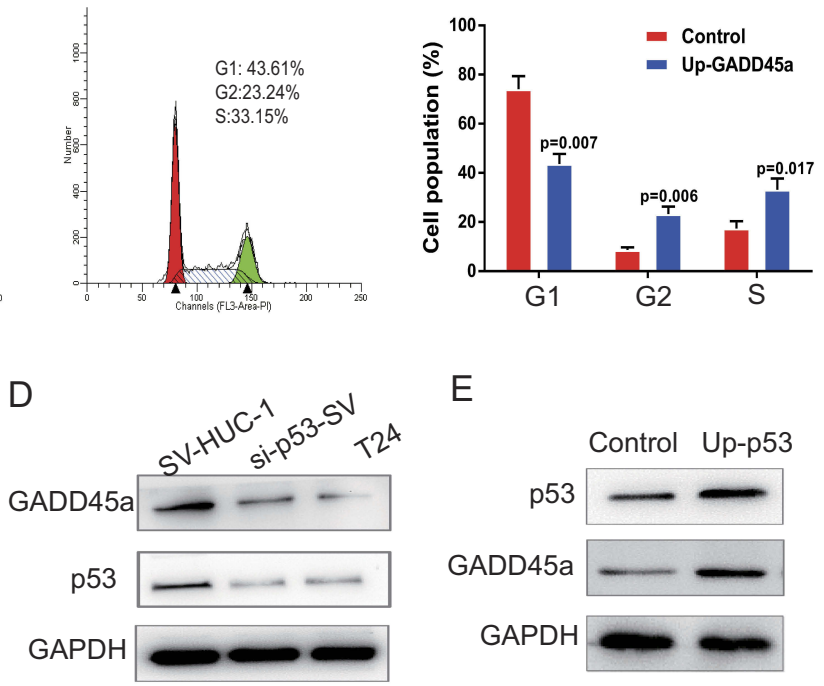

E

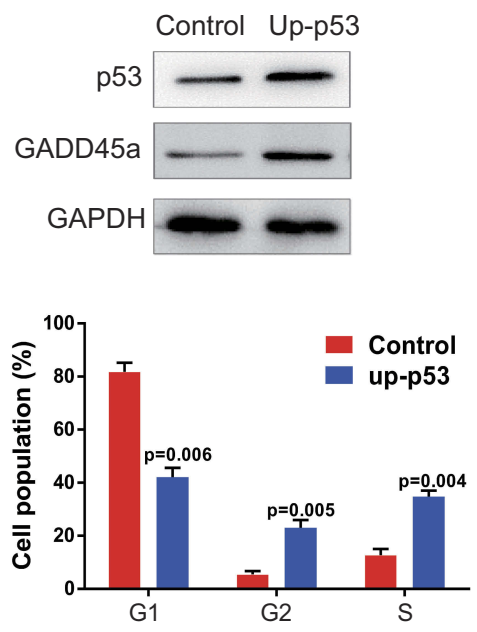

Figure 3 Upregulation of GADD45a delays BC cell cycle progression in the G2/M and S phase. (A) Flow cytometry analysis in T24 and 5637 cells showed that upregulation of GADD45a inhibited cell cycle progression in G2/M and $S$ phase. Histograms display the mean number of colonies, and the number of colonies was shown as the mean \pm $\mathrm{SD}$ of three independent experiments, $\mathrm{P}<0.05$ represents significance. (B) Western blot result showed that cdc2 and cyclinBI were upregulated in highly expressing GADD45a cells, whereas CDK2, CDK4 and cyclinDI were unchanged. (C) Western blotting showed that GADD45a expression was higher in normal urothelial SV-HUC-I cells than in T24 cells. (D) Knockdown of p53 in SV-HUC-I cells decreases GADD45a expression. (E) Upregulating p53 expression elevated GADD45a protein expression in T24 cells. (F) Upregulation of P53 inhibited T24 cell cycle progression in G2/M and S phase. Histograms display the mean number of colonies, and the number of colonies was shown as the mean \pm SD of three independent experiments, $P<0.05$ represents significance.

progression. ${ }^{3}$ Thus, it is meaningful to study GADD45a for BC treatment.

GADD45a was the first identified member of the GADD45 family, which contains two other members GADD45b and GADD45y. ${ }^{4}$ The GADD45 family was the 45th member of a collection of cDNA clones after cellular stress stimuli such as ultraviolet radiation as well as other growth cessation signals. All three members shared highly conserved sequence homology and were implicated in similar cellular responses often associated with stress signaling and other growth regulatory pathways. ${ }^{15}$ GADD45a was first reported and described as a stress-induced protein associated with growth arrest and stimulation of DNA-repair, as well as apoptosis and cell cycle inhibition. ${ }^{6,16}$ GADD45a implicated regulatory pathways are complicated and broad, with $\mathrm{p} 38$ and JNK stress mitogen-activated protein kinases (MAPK) as well as BRCA1, FOXOA3 and ATF4 being involved in the regulation of GADD45a expression. ${ }^{6}$ In the cellular nucleus, GADD45a protein interacts with a variety of proteins such as cdc2 and cdc2-cyclinB1 complex subsequently, 
A

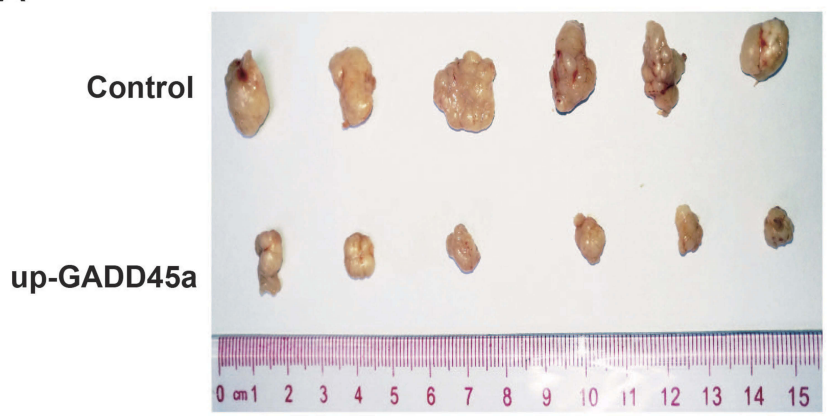

B

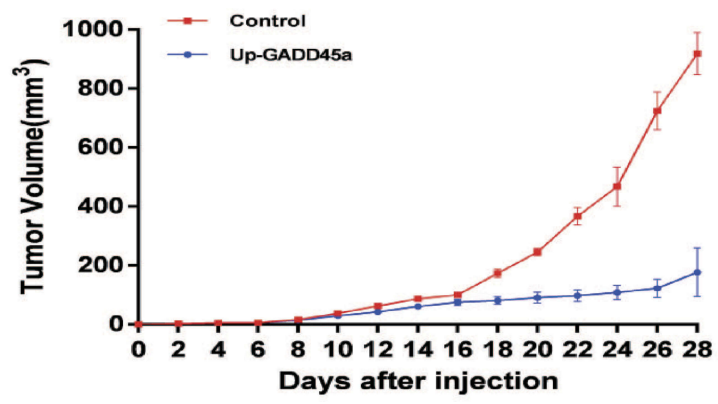

E
C

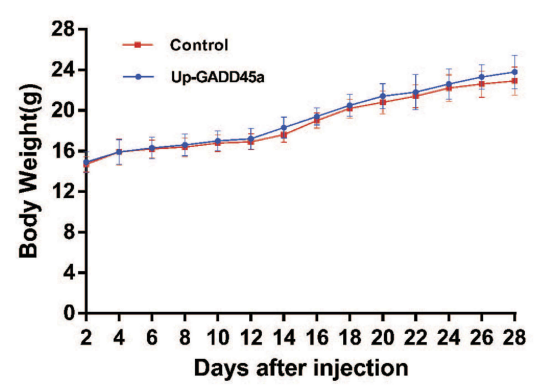

D

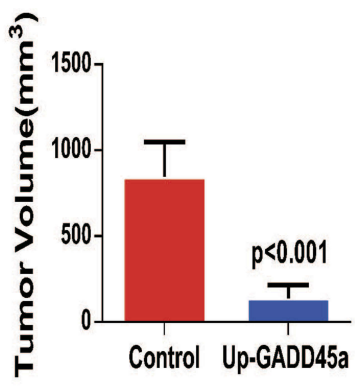

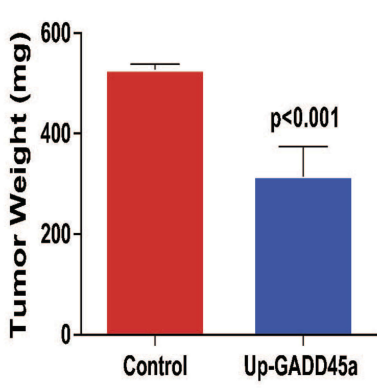

Figure 4 Upregulation of GADD45a inhibits BC tumor growth in vivo. (A) The xenograft tumors were isolated from mice at the end of study. (B) Tumor volumes were recorded from the date of injection to the end of the study as shown by the dashed lines in the graphs $($ mean, $n=6)$. (C) Body weights were recorded from the date of injection to the end of the study as shown by the dashed lines in the graphs (mean, $n=6)$. (D) Tumor volumes were recorded at the end of the study ( $n=6$ ). (E) Histograms present the mean tumor weight in each group, means $\pm S D(n=6) . p<0.05$ represents significance.

participating in G2 checkpoint mechanisms and consequently inhibiting cell cycle progression in the $\mathrm{G} 2 / \mathrm{M}$ phase. ${ }^{9,10,17}$

Because GADD45a inhibits DNA-repaired cell proliferation, it is thought to be a tumor suppressor of human cancers including breast cancer, prostate cancer, and gastric cardia adenocarcinoma. ${ }^{18-21}$ It is reported that GADD45a is a direct target of the tumor suppressor FOXO3A, by which way that FOXO3a binds to the GADD45a promoter and induces GADD45a transcription. ${ }^{22}$ In addition, other tumor suppressor genes such as activating transcription factor-4 (ATF-4), APRIL and BRCA1 also play critical roles in GADD45amediated cell cycle inhibition. ${ }^{23-25}$

In this study, we detected the expression of GADD45a in $\mathrm{BC}$ tissues and found that GADD45a was downregulated in $\mathrm{BC}$ tissues and cells, suggesting potential suppression of GADD45a in BC development. This was validated later when we found that $\mathrm{BC}$ cell growth and proliferation were impaired when GADD45a expression increased. Thus, reducing GADD45a expression may contribute to $\mathrm{BC}$ development and progression.

GADD45a positively participates in the cell cycle checkpoint mechanism of cell cycle and it displaces
PCNA (proliferating cell nuclear antigen) from the cyclinD1 complex to delay the cell cycle in the $\mathrm{S}$ phase or in the G2/M phase by interacting with cdc2/ cyclinB1. ${ }^{13,26}$ Consistent with other studies, our results indicated that increasing GADD45a expression delayed the BC cell cycle in the $\mathrm{G} 2 / \mathrm{M}$ and $\mathrm{S}$ phases, accompanied with a reduction in $c$ c $2 /$ cyclinB1 activities.

P53 is a well-known and widely inactivated protein in many phenotypes of tumors, and it positively regulates GADD45a expression and GADD45a-mediated cell cycle inhibition. In addition, the p53-mediated GADD45a cell cycle regulation requires $\mathrm{p} 38$ activation by directly phosphorylating p53's Ser46 site. ${ }^{27,28}$ In wild BC cells, p53 was downregulated. However, when p53 expression increased, GADD45a expression was upregulated and the BC cell cycle was subsequently delayed in the $\mathrm{G} 2 / \mathrm{M}$ and $\mathrm{S}$ phase. This result supports that GADD45a-mediated cell cycle was p53regulated.

\section{Ethical Approval}

Our study was approved by the Ethics Committee of Sun Yat-Sen University Cancer Center, Guangzhou, China. 


\section{Conclusion}

In this study, we provided basic evidence that GADD45a was downregulated in BC, and GADD45a functions as a suppressor in $\mathrm{BC}$ progression by delaying cell cycle progression in a p53-regulated manner. Our study also suggested that GADD45a might become a potential target for treating $\mathrm{BC}$ in future.

\section{Acknowledgments}

This work was supported by grants from the Natural Science Foundation of Chongqing (No. cstc2019jcyj-msxmX0420), the Sun Yat-sen University Cancer Center Medical Scientist Training Program (No. 14zxqk08), the Chongqing Municipal Science and Technology Bureau and Health Bureau Joint Medical Research Project (No. 2018MSXM033), and the Performance Incentive and Guidance Program of Chongqing Scientific Research Institutions (cstc2017jxj1130012).

\section{Disclosure}

The authors report no conflicts of interest in this work.

\section{References}

1. Chen W, Zheng R, Baade PD, et al. Cancer statistics in China, 2015. CA Cancer J Clin. 2016;66(2):115-132. doi:10.3322/caac.21338

2. Siegel R, Ma J, Zou Z, Jemal A. Cancer statistics, 2014. CA Cancer J Clin. 2014;64(1):9-29. doi:10.3322/caac.21208

3. Yuan G, Chen X, Liu Z, et al. Flavagline analog FL3 induces cell cycle arrest in urothelial carcinoma cell of the bladder by inhibiting the Akt/PHB interaction to activate the GADD45 $\alpha$ pathway. $J$ Exp Clin Cancer Res. 2018;37(1):21. doi:10.1186/s13046-018-0695-5

4. Fornace AJ, Nebert DW, Hollander MC, et al. Mammalian genes coordinately regulated by growth arrest signals and DNA-damaging agents. Mol Cell Biol. 1989;9(10):4196-4203. doi:10.1128/ mcb.9.10.4196

5. Abdollahi A, Lord KA, Hoffman-Liebermann B, Liebermann DA. Sequence and expression of a cDNA encoding MyD118: a novel myeloid differentiation primary response gene induced by multiple cytokines. Oncogene. 1991;6(1):165-167.

6. Salvador JM, Brown-Clay JD, Fornace AJ. Gadd45 in stress signaling, cell cycle control, and apoptosis. Adv Exp Med Biol. 2013;793:119. doi:10.1007/978-1-4614-8289-5_1

7. Beadling C, Johnson KW, Smith KA. Isolation of interleukin 2induced immediate-early genes. Proc Natl Acad Sci U S A. 1993;90 (7):2719-2723. doi:10.1073/pnas.90.7.2719

8. Malumbres M, Barbacid M. Cell cycle, CDKs and cancer: a changing paradigm. Nat Rev Cancer. 2009;9(3):153-166. doi:10.1038/nrc2602

9. Wang XW, Zhan Q, Coursen JD, et al. GADD45 induction of a G2/M cell cycle checkpoint. Proc Natl Acad Sci U S A. 1999;96(7):37063711. doi:10.1073/pnas.96.7.3706

10. Zhan Q, Antinore MJ, Wang XW, et al. Association with Cdc2 and inhibition of $\mathrm{Cdc} 2 /$ cyclin $\mathrm{B} 1$ kinase activity by the p53-regulated protein Gadd45. Oncogene. 1999;18(18):2892-2900. doi:10.1038/sj. onc. 1202667
11. Hollander MC, Philburn RT, Patterson AD, et al. Deletion of XPC leads to lung tumors in mice and is associated with early events in human lung carcinogenesis. Proc Natl Acad Sci U S A. 2005;102 (37):13200-13205. doi:10.1073/pnas.0503133102

12. Yang F, Zhang W, Li D, Zhan Q. Gadd45a suppresses tumor angiogenesis via inhibition of the mTOR/STAT3 protein pathway. $J$ Biol Chem. 2013;288(9):6552-6560. doi:10.1074/jbc.M112.418335

13. Hollander MC, Fornace AJ. Genomic instability, centrosome amplification, cell cycle checkpoints and Gadd45a. Oncogene. 2002;21 (40):6228-6233. doi:10.1038/sj.onc. 1205774

14. Smith ML, Fornace AJ. Mammalian DNA damage-inducible genes associated with growth arrest and apoptosis. Mutat Res. 1996;340(23):109-124. doi:10.1016/s0165-1110(96)90043-3

15. Gao M, Guo N, Huang C, Song L. Diverse roles of GADD45alpha in stress signaling. Curr Protein Pept Sci. 2009;10(4):388-394.

16. Cretu A, Sha X, Tront J, Hoffman B, Liebermann DA. Stress sensor Gadd45 genes as therapeutic targets in cancer. Cancer Ther. 2009;7 (A):268-276.

17. Paruthiyil S, Cvoro A, Tagliaferri M, Cohen I, Shtivelman E, Leitman DC. Estrogen receptor $\beta$ causes a G2 cell cycle arrest by inhibiting CDK1 activity through the regulation of cyclin B1, GADD45A, and BTG2. Breast Cancer Res Treat. 2011;129(3):777784. doi:10.1007/s10549-010-1273-5

18. Wang W, Huper G, Guo Y, Murphy SK, Olson JA, Marks JR. Analysis of methylation-sensitive transcriptome identifies GADD45a as a frequently methylated gene in breast cancer. Oncogene. 2005;24(16):2705-2714. doi:10.1038/sj.onc. 1208464

19. Ramachandran K, Gopisetty G, Gordian E, et al. Methylationmediated repression of GADD45alpha in prostate cancer and its role as a potential therapeutic target. Cancer Res. 2009;69(4):15271535. doi:10.1158/0008-5472.CAN-08-3609

20. Guo W, Dong Z, Guo Y, Chen Z, Kuang G, Yang Z. Methylationmediated repression of GADD45A and GADD45G expression in gastric cardia adenocarcinoma. Int J Cancer. 2013;133(9):20432053. doi:10.1002/ijc. 28223

21. Schneider G, Weber A, Zechner U, et al. GADD45alpha is highly expressed in pancreatic ductal adenocarcinoma cells and required for tumor cell viability. Int $J$ Cancer. 2006;118(10):2405-2411. doi: $10.1002 / \mathrm{ijc} .21637$

22. Tran H, Brunet A, Grenier JM, et al. DNA repair pathway stimulated by the forkhead transcription factor FOXO3a through the Gadd45 protein. Science. 2002;296(5567):530-534. doi:10.1126/science.1068712

23. Notas G, Alexaki VI, Kampa M, et al. APRIL binding to BCMA activates a JNK2-FOXO3-GADD45 pathway and induces a G2/M cell growth arrest in liver cells. J Immunol. 2012;189(10):4748-4758. doi:10.4049/jimmunol.1102891

24. Li S, Ting NS, Zheng L, et al. Functional link of BRCA1 and ataxia telangiectasia gene product in DNA damage response. Nature. 2000;406(6792):210-215. doi:10.1038/35018134

25. Hartman AR, Ford JM. BRCA1 induces DNA damage recognition factors and enhances nucleotide excision repair. Nat Genet. 2002;32 (1):180-184. doi:10.1038/ng953

26. Smith ML, Chen IT, Zhan Q, et al. Interaction of the p53-regulated protein Gadd45 with proliferating cell nuclear antigen. Science. 1994;266(5189):1376-1380. doi:10.1126/science.7973727

27. Hildesheim J, Fornace AJ. The dark side of light: the damaging effects of UV rays and the protective efforts of MAP kinase signaling in the epidermis. DNA Repair (Amst). 2004;3(6):567-580. doi:10.1016/j.dnarep.2004.02.012

28. Bulavin DV, Kovalsky O, Hollander MC, Fornace AJ. Loss of oncogenic $\mathrm{H}$-ras-induced cell cycle arrest and p38 mitogen-activated protein kinase activation by disruption of Gadd45a. Mol Cell Biol. 2003;23(11):3859-3871. doi:10.1128/mcb.23.11.3859-3871.2003 


\section{Publish your work in this journal}

OncoTargets and Therapy is an international, peer-reviewed, open access journal focusing on the pathological basis of all cancers, potential targets for therapy and treatment protocols employed to improve the management of cancer patients. The journal also focuses on the impact of management programs and new therapeutic agents and protocols on patient perspectives such as quality of life, adherence and satisfaction. The manuscript management system is completely online and includes a very quick and fair peer-review system, which is all easy to use. Visit http://www.dovepress.com/ testimonials.php to read real quotes from published authors.

Submit your manuscript here: https://www.dovepress.com/oncotargets-and-therapy-journal 